\title{
Higher regularity for solutions to elliptic systems in divergence form subject to mixed boundary conditions
}

\author{
Robert Haller-Dintelmann ${ }^{1} \cdot$ Hannes Meinlschmidt ${ }^{2}$ (D) Winnifried Wollner $^{3}$
}

Received: 5 July 2018 / Accepted: 17 December 2018 / Published online: 26 December 2018

(c) Fondazione Annali di Matematica Pura ed Applicata and Springer-Verlag GmbH Germany, part of Springer Nature 2018

\begin{abstract}
This work combines results from operator and interpolation theory to show that elliptic systems in divergence form admit maximal elliptic regularity on the Bessel potential scale $H_{D}^{s-1}(\Omega)$ for $s>0$ sufficiently small, if the coefficient in the main part satisfies a certain multiplier property on the spaces $H^{s}(\Omega)$. Ellipticity is enforced by assuming a Gårding inequality, and the result is established for spaces incorporating mixed boundary conditions with very low regularity requirements for the underlying spatial set. To illustrate the applicability of our results, two examples are provided. Firstly, a phase-field damage model is given as a practical application where higher differentiability results are obtained as a consequence to our findings. These are necessary to show an improved numerical approximation rate. Secondly, it is shown how the maximal elliptic regularity result can be used in the context of quasilinear parabolic equations incorporating quadratic gradient terms.
\end{abstract}

Keywords Maximal elliptic regularity $\cdot$ Non-Lipschitz coefficients $\cdot$ Second-order divergence operators · Elliptic system · Mixed boundary conditions · Phase-field damage

Mathematics Subject Classification Primary 35B65; Secondary 35J57 · 35J25

Hannes Meinlschmidt

hannes.meinlschmidt@ricam.oeaw.ac.at

Robert Haller-Dintelmann

haller@mathematik.tu-darmstadt.de

Winnifried Wollner

wollner@mathematik.tu-darmstadt.de

1 Fachbereich Mathematik, Technische Universität Darmstadt, Schloßgartenstraße 7, 64285

Darmstadt, Germany

2 Johann Radon Institute for Computational and Applied Mathematics (RICAM), Altenberger Straße 69, 4040 Linz, Austria

3 Fachbereich Mathematik, Technische Universität Darmstadt, Dolivostraße 15, 64293 Darmstadt, Germany 


\section{Introduction}

In this paper, we consider the weak form of a system of elliptic $(n \times n)$ operators $\mathbb{A}$ in divergence form induced by the tensor $A$ for a function $u: \Omega \rightarrow \mathbb{C}^{n}\left(\right.$ or $\left.\mathbb{R}^{n}\right)$ :

$$
\begin{aligned}
& (\mathbb{A} u)_{1}=-\sum_{\alpha, \beta=1}^{d} \sum_{j=1}^{n} \partial_{\alpha}\left(A_{\alpha, \beta}^{1, j} \partial_{\beta} u_{j}\right) \\
& \text { : } \vdots \quad \vdots \\
& (\mathbb{A} u)_{n}=-\sum_{\alpha, \beta=1}^{d} \sum_{j=1}^{n} \partial_{\alpha}\left(A_{\alpha, \beta}^{n, j} \partial_{\beta} u_{j}\right) .
\end{aligned}
$$

Each equation, for $j \in\{1, \ldots, n\}$, in this system is subject to possibly mixed boundary conditions with a Dirichlet boundary part $D_{j}$ and we set $D:=\times_{j=1}^{n} D_{j}$. Precise assumptions on $A$, the domain $\Omega$, and the Dirichlet boundary parts $D_{j}$ are given below in Assumptions 1 and 2 .

As a motivation for our results, let us assume for the moment that the operator $\mathbb{A}$ defines an isomorphism between the Sobolev Hilbert spaces $\mathbb{H}_{D}^{1}(\Omega) \rightarrow \mathbb{H}_{D}^{-1}(\Omega)$, as it can be asserted by the notorious Lax-Milgram theorem in many cases. It is known under mild assumptions on $\Omega$ and $D$ that if $A_{\alpha, \beta}^{i, j} \in L^{\infty}(\Omega)$, then $\mathbb{A}$ remains an isomorphism for small perturbations in the integrability scale, i.e., there exists $\varepsilon>0$ such that the mapping

$$
\mathbb{A}: \mathbb{H}_{D}^{1, p}(\Omega) \rightarrow \mathbb{H}_{D}^{-1, p}(\Omega)
$$

remains an isomorphism for all $p$ satisfying $2-\varepsilon \leq p \leq 2+\varepsilon$, see, e.g., [13,14] or [15].

We will show that such a result is also true on the differentiability scale: Indeed, we prove that if $A$ is a multiplier on $\mathbb{H}^{\varepsilon}$ for some $0<\varepsilon<\frac{1}{2}$, then there exists $\vartheta>0$ such that the mapping

$$
\mathbb{A}: \mathbb{H}_{D}^{1+\theta}(\Omega) \rightarrow \mathbb{H}_{D}^{\theta-1}(\Omega)
$$

is still an isomorphism for any $\theta$ satisfying $-\vartheta \leq \theta \leq \vartheta$. Moreover, the norm of the inverse of $\mathbb{A}$ is uniform in its coercivity constant and the bound on the multiplier norm; in particular, it does not depend explicitly on the actual multiplier at hand. We note that the required multiplier property for $A$ is in particular satisfied if $A$ is $\sigma$-Hölder continuous for $\sigma>\varepsilon$ or if $A$ is a characteristic function of an open subset of $\Omega$ with locally finite perimeter. Similar results have been obtained by Jochmann in [17] for the case of a scalar elliptic problem on a domain with a piecewise smooth boundary. Our work can therefore be seen as an extension of these results to elliptic systems, while allowing much less regular geometries for $\Omega$ and the boundary parts $D_{j}$. The authors are not aware of a similar result so far which incorporates both systems and fully non-smooth geometry.

Results as described above are interesting, firstly because they provide a sharp maximal elliptic regularity result for the abstract equation $\mathbb{A} u=f$ in $\mathbb{H}_{D}^{\theta-1}(\Omega)$. This allows for instance to obtain certain improved convergence rates in the analysis of $\mathbb{H}^{1}$-discretization errors for such equations, which is possible only if a gap in differentiability is present, see our example in Sect. 7. Further, the fact that the norm of the inverse of $\mathbb{A}$ is uniform for all multipliers with a certain coercivity constant and multiplier norm makes the result also attractive to use in a nonlinear elliptic setting via fixed-point techniques. Finally, the knowledge that $\mathbb{H}_{D}^{1+\theta}(\Omega)$ is the domain of $\mathbb{A}$ over $\mathbb{H}_{D}^{\theta-1}(\Omega)$ for all coefficient functions in 
a certain class is a very valuable information when aiming to treat non-autonomous timedependent problems of the form

$$
u^{\prime}(t)+\mathbb{A}(t) u(t)=f(t) \quad \text { in } \mathbb{H}_{D}^{\theta-1}(\Omega), \quad u(0)=u_{0},
$$

see, e.g., $[1,26]$, or even for quasilinear equations such as

$$
u^{\prime}(t)+\mathbb{A}(u(t)) u(t)=F(u(t)) \text { in } \mathbb{H}_{D}^{\theta-1}(\Omega), \quad u(0)=u_{0} .
$$

We explain the latter in some more detail in Sect. 8. Here, the advantage of the improved differentiability scale instead of the integrability one is that the former allows to treat quadratic terms in $F$, so $F(u(t))=|\nabla u(t)|^{2}$, at least in space dimension $d=2$, which are known to be notoriously difficult to handle.

Throughout the paper, the considered Banach spaces are in general complex vector spaces. $\mathrm{By} \cong$ we understand that two normed spaces are equal up to equivalent norms. Moreover, the restriction of $f: U \rightarrow \mathbb{C}$ to $\Lambda \subseteq U$ will be denoted by $f_{\uparrow \Lambda}$ and we use $B_{r}(x)$ for the ball of radius $r$ around $x$ in $\mathbb{R}^{d}$.

The rest of the paper is structured as follows: We will start by stating our main result in Sect. 2 together with the notation of the subsequent sections. In Sect. 3, we will give the details on the assumed regularity of the domain: We assume that (the closure of) the nonDirichlet boundary parts admit bi-Lipschitz boundary charts and allow the Dirichlet parts of the domain to be $(d-1)$-sets. In Sect. 4, we will define the Bessel potential function spaces needed in the statement of our result. The collection of preliminaries ends in Sect. 5, where we briefly introduce the concept of a multiplier space and provide some more accessible examples for when a coefficient function is in fact a multiplier. After these preparations, we come to the proof of the main result in Sect. 6. We conclude the paper by applications of our results to a phase-field fracture/damage model in Sect. 7 and to quasilinear equations in Sect. 8.

\section{Main result}

We first give our main result. All occurring spaces and the notion of a multiplier are formally introduced and defined below (cf. Definitions 2, 3 and 4).

Assumption 1 For $i, j \in\{1, \ldots, n\}$, each matrix $A^{i, j}$ is a $(d \times d)$ matrix with $A_{\alpha, \beta}^{i, j} \in L^{\infty}(\Omega)$ for $\alpha, \beta \in\{1, \ldots, d\}$.

To formulate the weak form of the elliptic system operator (1), let

$$
\mathbb{H}_{D}^{1}(\Omega):=\prod_{j=1}^{n} H_{D_{j}}^{1}(\Omega),
$$

and let $\mathbb{H}_{D}^{-1}(\Omega)$ be the anti-dual space of $\mathbb{H}_{D}^{1}(\Omega)$. For a tensor $A$ satisfying Assumption 1 , we define the form $a: \mathbb{H}_{D}^{1}(\Omega) \times \mathbb{H}_{D}^{1}(\Omega) \rightarrow \mathbb{C}$ and the divergence-gradient system operator $-\nabla \cdot A \nabla: \mathbb{H}_{D}^{1}(\Omega) \rightarrow \mathbb{H}_{D}^{-1}(\Omega)$ by

$$
\begin{aligned}
\langle-\nabla \cdot A \nabla u, v\rangle & :=a(u, v) \\
& :=\sum_{i, j=1}^{n} \int_{\Omega}\left(A^{i, j} \nabla u_{j}\right) \cdot \nabla \overline{v_{i}} \mathrm{~d} x \text { for } u, v \in \mathbb{H}_{D}^{1}(\Omega) .
\end{aligned}
$$


We extend this slightly by defining $-\nabla \cdot A \nabla+\gamma: \mathbb{H}_{D}^{1}(\Omega) \rightarrow \mathbb{H}_{D}^{-1}(\Omega)$ for $\gamma \geq 0$ by

$$
\langle(-\nabla \cdot A \nabla+\gamma) u, v\rangle:=\langle-\nabla \cdot A \nabla u, v\rangle+\sum_{j=1}^{n} \int_{\Omega} \gamma u_{j} \overline{v_{j}} \mathrm{~d} x
$$

and formulate our main result as follows:

Theorem 1 Let Assumptions 1 and 2 be satisfied and suppose that the system (1) is elliptic in the sense that it satisfies $a$ Gårding inequality, i.e., there exist $\lambda>0$ and $\mu \geq 0$ such that

$$
\operatorname{Re}(a(u, u)) \geq \sum_{i=1}^{n} \lambda\left\|\nabla u_{i}\right\|_{L^{2}\left(\Omega ; \mathbb{C}^{d}\right)}^{2}-\mu\left\|u_{i}\right\|_{L^{2}(\Omega)}^{2} \quad \text { for all } u \in \mathbb{H}_{D}^{1}(\Omega) .
$$

Assume further that each matrix $A^{i, j}$ is a multiplier on $H^{\varepsilon}(\Omega)^{d}$ for some $0<\varepsilon<\frac{1}{2}$. Then, there exist $\gamma \geq 0$ and $0<\delta \leq \varepsilon$ such that

$$
-\nabla \cdot A \nabla+\gamma \in \mathcal{L}_{\text {iso }}\left(\mathbb{H}_{D}^{\theta+1}(\Omega) ; \mathbb{H}_{D}^{\theta-1}(\Omega)\right) \text { for all }|\theta|<\delta,
$$

i.e., $-\nabla \cdot A \nabla+\gamma$ is a topological isomorphism between $\mathbb{H}_{D}^{\theta+1}(\Omega)$ and $\mathbb{H}_{D}^{\theta-1}(\Omega)$ for every $-\delta<\theta<\delta$. Both the size of $\delta$ and the norm of the inverse of $-\nabla \cdot A \nabla+\gamma$ are uniformly bounded w.r.t. the multiplier norm of $A$, the coefficients in the Gårding inequality and $\gamma$.

Remark 1 (i) The need for the perturbation $\gamma \geq 0$ in Theorem 1 is due to the possibility that 0 might be an eigenvalue of $\mathbb{A}$. If this is not the case, $\gamma=0$ can be chosen. In particular, $\gamma=0$ is allowed if $\mu=0$ and if a Poincaré inequality holds true for $\mathbb{H}_{D}^{1}(\Omega)$. The latter is already satisfied for $D \neq \varnothing$ in our geometric setting as given in Sect. 3 below, cf. [3, Rem. 3.4].

(ii) We give sufficient conditions for the matrix functions $A^{i, j}$ to be multipliers on $H^{\varepsilon}(\Omega)^{d}$ in Lemma 1 below. A particular case is when $A_{\alpha \beta}^{i, j} \in \mathrm{C}^{\sigma}(\Omega)$ for some $\varepsilon<\sigma<1$ and all $\alpha, \beta \in\{1, \ldots, d\}$, where $\mathrm{C}^{\sigma}(\Omega)$ is the space of $\sigma$-Hölder continuous functions on $\Omega$. This also implies that $\mathrm{C}^{\frac{1}{2}}(\Omega)$ is always a suitable multiplier space for Theorem 1 . Note, however, that a multiplier need not necessarily be continuous, see Remark 5 .

(iii) The Gårding inequality as the abstract tool to enforce coercivity in our context goes back to [24]. We consider it as the adequate concept here because of its generality, although it is not easy to give weak conditions on the coefficient tensor $A$ which are sufficient for the Garrding inequality to hold. For the pure Dirichlet case, so $D_{j}=\partial \Omega$ for all $j=1, \ldots, n$, it is known that the Garrding inequality holds true if $A$ satisfies the Legendre-Hadamard condition and the coefficient functions are uniformly continuous, see [10, Ch. 3.4.3]. (Hölder functions as multipliers would thus be admissible.) On the other hand, in the pure Neumann case $D=\emptyset$, the Legendre-Hadamard condition is in general not sufficient even in the case of constant coefficients, as the counterexample $a(u, u)=\int_{\Omega}|\bar{\partial} u|^{2} \mathrm{~d} x$ with the Cauchy-Riemann operator $\bar{\partial}$ shows. ${ }^{1}$ Other than a very strong ellipticity assumption in the form of a Legendre condition, a general condition on $A$ for the case of non-Dirichlet data, so $D_{j} \neq \partial \Omega$, seems unknown, although there are some particular positive results. We refer to $[28,32]$ and the references therein. Let us also mention the connection to Korn's inequality, see, e.g., [7,16] or [22] and the references there.

Theorem 1 yields the following corollary:

\footnotetext{
${ }^{1}$ We thank the anonymous referee for this simple but instructive example.
} 
Corollary 1 In the situation of Theorem 1 , let $f \in \mathbb{H}_{D}^{\theta-1}(\Omega)$ for some $0<\theta<\delta$. Then, the elliptic system

$$
-\nabla \cdot A \nabla u+\gamma u=f \quad \text { in } \mathbb{H}_{D}^{\theta-1}(\Omega)
$$

has a unique solution $u \in \mathbb{H}_{D}^{\theta+1}(\Omega)$ satisfying

$$
\|u\|_{\mathbb{H}_{D}^{\theta+1}(\Omega)} \leq C\|f\|_{\mathbb{H}_{D}^{\theta-1}(\Omega)}
$$

for some constant $C \geq 0$ independent of $f$ and uniform in the multiplier norm of $A$, the constants in the Gårding inequality and $\gamma$. Moreover, for all $0<\eta<\theta$ there exist $p>2$ and $C^{\bullet} \geq 0$ such that $u \in \mathbb{H}_{D}^{1+\eta, p}(\Omega)$ and

$$
\|u\|_{\mathbb{H}_{D}^{1+\eta, p}(\Omega)} \leq C^{\bullet}\|f\|_{\mathbb{H}_{D}^{\theta-1}(\Omega)}
$$

where $C^{\bullet}$ is uniform in the same quantities as $C$ is.

Remark 2 A particular case for $f=\left(f_{1}, \ldots, f_{n}\right)$ being in $\mathbb{H}_{D}^{\theta-1}(\Omega)$ is when we have $f_{j} \in$ $L^{q_{j}}(\Omega)$ for $q_{j} \geq \frac{2 d}{d+2(1-\theta)}$. This follows from the embedding

$$
L^{q_{j}}(\Omega) \hookrightarrow H_{D_{j}}^{\theta-1}(\Omega) \quad \text { if } q_{j} \geq \frac{2 d}{d+2(1-\theta)}
$$

which can be inferred from the extension property for $H_{D_{j}}^{1-\theta}(\Omega)$ as in [9, Thm. 5.1], the embedding $H^{1-\theta}\left(\mathbb{R}^{n}\right) \hookrightarrow L^{q_{j}^{\prime}}\left(\mathbb{R}^{n}\right)$ in $\mathbb{R}^{n}[29$, Ch. 2.8.1], and a duality argument; see Sect. 4 for the formal definitions of the function spaces.

\section{Assumptions on the domain}

We formulate the assumptions on the spatial domain $\Omega \subset \mathbb{R}^{d}$ and its boundary. As part of the assumptions on Theorem 1, these are supposed to be valid in all of the following. A preliminary definition we need is the following (cf. [18]):

Definition $1((d-1)$-set $)$ Let $F \subset \mathbb{R}^{d}$ be a Borel set. We say that $F$ is a $(d-1)$-set or that $F$ satisfies the Ahlfors-David condition if there is $c \geq 1$ such that

$$
c^{-1} r^{d-1} \leq \mathcal{H}^{d-1}\left(F \cap B_{r}(x)\right) \leq c r^{d-1} \text { for all } x \in F, 0<r \leq 1,
$$

where $\mathcal{H}^{d-1}$ is the $(d-1)$-dimensional Hausdorff measure.

The assumptions on $\Omega$ and $D_{j}$ for $j \in\{1, \ldots, n\}$ are then as follows, where we set $\mathfrak{D}:=\bigcap_{j=1}^{n} D_{j}:$

Assumption 2 The set $\Omega \subset \mathbb{R}^{d}$ is a bounded domain and for each $j \in\{1, \ldots, n\}$, the set $D_{j} \subseteq \partial \Omega$ is either empty or a closed $(d-1)$-set. For every point $x \in \overline{\partial \Omega \backslash \mathfrak{D}}$, there are Lipschitz boundary charts available; that is, there exists an open neighborhood $U_{x}$ of $x$ and a bi-Lipschitz map $\phi_{x}: U_{x} \rightarrow(-1,1)^{d}$ such that $\phi_{x}(x)=0$ and

$$
\begin{aligned}
\phi_{x}\left(U_{x} \cap \Omega\right) & =\left\{x \in(-1,1)^{d}: x_{d}<0\right\}, \\
\phi_{x}\left(U_{x} \cap \partial \Omega\right) & =\left\{x \in(-1,1)^{d}: x_{d}=0\right\} .
\end{aligned}
$$




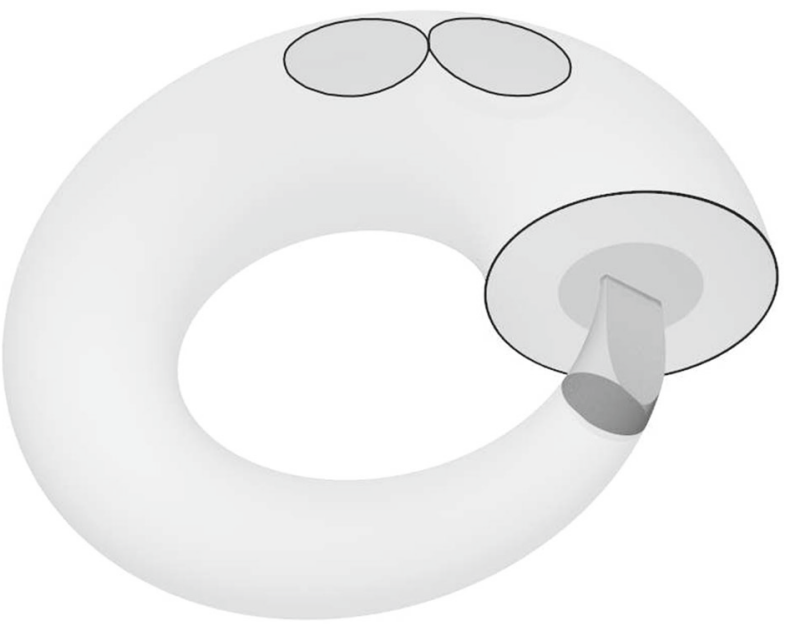

Fig. 1 Example of a domain $\Omega$ which is admissible under Assumption 2. The areas at the boundary shaded in dark carry Dirichlet conditions

We refer to Figure 1 for an example of the admissible geometry in Assumption 2, a chisel bent onto itself, with Dirichlet boundary data at the darker areas. At the contact area of the top of the chisel with its base, which is part of the Dirichlet boundary, $\Omega$ lies at both sides of its boundary. Moreover, the contact area is allowed to be degenerated to a line segment there.

Remark 3 (i) For $\mathfrak{D}=\emptyset$, the assumptions on $\Omega$ fall back to that of a classical Lipschitz domain (cf. [12]). On the other side of the spectrum, for $\mathfrak{D}=\partial \Omega$, so pure Dirichlet conditions for every equation in the system (1), we do not require local descriptions of $\partial \Omega$ by boundary charts at all.

(ii) If $\Omega \cup D_{j}$ is regular in the sense of Gröger (cf. [13,14]) for some $j \in\{1, \ldots, n\}$, then Assumption 2 is already satisfied. Indeed, in this case $D_{j}$ is already a $(d-1)$-set, and there are already bi-Lipschitz charts available for the whole $\partial \Omega$, so $\Omega$ is again a Lipschitz domain. This follows from the facts that the concept of Gröger requires that $D_{j} \supseteq \mathfrak{D}$ is also described by local bi-Lipschitz charts as $\overline{\partial \Omega \backslash \mathfrak{D}}$ is in Assumption 2, that such a local bi-Lipschitz description of $D_{j}$ implies that $D_{j}$ is a $(d-1)$-set by [18, Ch. II.1.1, Ex. 1], and that finite unions of $(d-1)$-sets are again $(d-1)$-sets. Clearly, Assumption 2 is also satisfied if $\Omega \cup D_{j}$ is regular in the sense of Gröger for every $j \in\{1, \ldots, n\}$.

(iii) With the same argument as in the previous point, we find that under Assumption 2, the whole boundary $\partial \Omega$ is always a $(d-1)$-set.

\section{Definitions and basics}

We move to exact definitions of the fundamental function spaces. Here, we mostly work only with the scalar-valued spaces $H_{F}^{s, p}(\Omega)$ for $(d-1)$-sets $F$ satisfying $\mathfrak{D} \subseteq F \subseteq \partial \Omega$ since their properties translate to $n$-fold products of such spaces immediately. Note that under Assumption 2, every $D_{j}$ is a valid choice for such $F$, as is $\partial \Omega$ by Remark 3 (iii). 
Definition 2 (Bessel potential spaces) Let $1<p<\infty$ and $\frac{1}{p}<s<1+\frac{1}{p}$, and consider a $(d-1)$-set $F$ such that $\mathfrak{D} \subseteq F \subseteq \partial \Omega$. Denote by $H^{s, p}\left(\mathbb{R}^{d}\right)$ the classical Bessel potential spaces, cf. [29, Ch. 2.3.1/Thm. 2.3.3]. Then, we make the following definitions:

(i) Set

$$
\begin{aligned}
& H_{F}^{s, p}\left(\mathbb{R}^{d}\right):=\left\{f \in H^{s, p}\left(\mathbb{R}^{d}\right):\right. \\
& \left.\quad \lim _{r \searrow 0} \frac{1}{\left|B_{r}(x)\right|} \int_{B_{r}(x)} f(y) \mathrm{d} y=0 \text { for } \mathcal{H}^{d-1} \text {-a.e. } x \in F\right\}
\end{aligned}
$$

with $H_{F}^{s}\left(\mathbb{R}^{d}\right):=H_{F}^{s, 2}\left(\mathbb{R}^{d}\right)$ and $\|\cdot\|_{H_{F}^{s, p}\left(\mathbb{R}^{d}\right)}=\|\cdot\|_{H^{s, p}\left(\mathbb{R}^{d}\right)}$.

(ii) Further, set $H_{F}^{s, p}(\Omega):=\left\{f_{\uparrow \Omega}: f \in H_{F}^{s, p}\left(\mathbb{R}^{d}\right)\right\}$, equipped with the factor space norm

$$
\|f\|_{H_{F}^{s, p}(\Omega)}:=\inf \left\{\|g\|_{H^{s, p}\left(\mathbb{R}^{d}\right)}: g \in H_{F}^{s, p}\left(\mathbb{R}^{d}\right), g_{\mid \Omega}=f\right\} .
$$

We set, again, $H_{F}^{s}(\Omega):=H_{F}^{s, 2}(\Omega)$, and for $F=\emptyset$, we write $H^{s, p}(\Omega):=H_{\emptyset}^{s, p}(\Omega)$.

(iii) Denote by $H_{F}^{-s}\left(\mathbb{R}^{d}\right)$ and $H_{F}^{-s}(\Omega)$ the space of antilinear continuous functionals acting on $H_{F}^{s}\left(\mathbb{R}^{d}\right)$ and $H_{F}^{s}(\Omega)$, respectively. We agree that the convention $H^{-s}(\Omega):=$ $H_{\emptyset}^{-s}(\Omega)$ still applies.

(iv) For $\Lambda \in\left\{\Omega, \mathbb{R}^{d}\right\}$ and $D_{j}$ from Assumption 2 , set $\mathbb{H}_{D}^{s, p}(\Lambda):=\prod_{j=1}^{n} H_{D_{j}}^{s, p}(\Lambda)$, with all the previous conventions for $p=2$, and let $\mathbb{H}_{D}^{-s}(\Lambda)$ be the space of continuous antilinear functionals on $\mathbb{H}_{D}^{s}(\Lambda)$, so $\mathbb{H}_{D}^{-s}(\Lambda):=\prod_{j=1}^{n} H_{D_{j}}^{-s}(\Lambda)$.

Remark 4 (i) For $1 \leq s<1+\frac{1}{p}$, it is easy to see that $H_{F}^{s, p}\left(\mathbb{R}^{d}\right)=H_{F}^{1, p}\left(\mathbb{R}^{d}\right) \cap H^{s, p}\left(\mathbb{R}^{d}\right)$ and that accordingly $H_{F}^{s, p}(\Omega) \subseteq H_{F}^{1, p}(\Omega) \cap H^{s, p}(\Omega)$. If there exists an extension operator $E$ which maps $H_{F}^{1, p}(\Omega)$ into $H_{F}^{1, p}\left(\mathbb{R}^{d}\right)$ and $H^{s, p}(\Omega)$ into $H^{s, p}\left(\mathbb{R}^{d}\right)$ at the same time such that $(E f)_{\mid \Omega}=f$, then the reverse inclusion and thus

$$
H_{F}^{s, p}(\Omega)=H_{F}^{1, p}(\Omega) \cap H^{s, p}(\Omega)
$$

follows. A particular case in which this extension property for $\Omega$ is satisfied is when $\Omega \cup$ $D_{j}$ is regular in the sense of Gröger for some $j \in\{1, \ldots, n\}$ (cf. Remark 3 (ii)) because then $\Omega$ is a Lipschitz domain for which the $H^{s, p}$-extension property is classical ([11, Thm. 7.25]), and the preservation of the zero trace on $F$ for the $H^{1, p}$-extension follows as in [8, Cor. 2.2.13].

(ii) Many authors commonly use $H_{0}^{s}(\Omega)$ instead of $H_{\partial \Omega}^{s}(\Omega)$ and $H^{-1}(\Omega)$ instead of $H_{\partial \Omega}^{-1}(\Omega)$. We feel that while this is adequate as long as only one fixed part of the boundary, e.g., $F=\partial \Omega$, is considered, a more careful notation is needed in view of the importance of both the sets $D_{j}$ and $\partial \Omega$.

The rather abstract definition of $H_{F}^{1}(\Omega)$ turns out to be equivalent to the classical Sobolev space with partially vanishing trace $\mathrm{W}_{F}^{1,2}(\Omega)$ which we formally define as follows.

Definition 3 (Sobolev spaces with partially vanishing trace) Let $F$ be a $(d-1)$-set satisfying $\mathfrak{D} \subseteq F \subseteq \partial \Omega$, and let $\Lambda \subseteq \mathbb{R}^{d}$ be a domain. Then, we set

$$
\mathrm{C}_{F}^{\infty}(\Lambda):=\left\{f_{\uparrow \Lambda}: f \in \mathrm{C}_{c}^{\infty}\left(\mathbb{R}^{d}\right), \operatorname{supp} f \cap F=\emptyset\right\}
$$


and

$$
\mathrm{W}_{F}^{1,2}(\Lambda):={\overline{\mathrm{C}_{F}^{\infty}(\Lambda)}}^{\|\cdot\|_{\mathrm{W}^{1,2}(\Lambda)}}
$$

for

$$
\|f\|_{\mathrm{W}^{1,2}(\Lambda)}:=\left(\int_{\Lambda}|f|^{2}+\|\nabla f\|_{2}^{2} \mathrm{~d} x\right)^{\frac{1}{2}} .
$$

Proposition 1 ([9, Cor. 3.8]) Let $F$ be a $(d-1)$-set satisfying $\mathfrak{D} \subseteq F \subseteq \partial \Omega$. Then, there holds $\mathrm{W}_{F}^{1,2}(\Omega) \cong H_{F}^{1}(\Omega)$.

Using Proposition 1 , we easily verify that $-\nabla \cdot A \nabla$ as in (2) is indeed well defined as an operator from $\mathbb{H}_{D}^{1}(\Omega)$ to $\mathbb{H}_{D}^{-1}(\Omega)$ under Assumption 1 .

\section{Multipliers}

We finally turn to the notion of a multiplier. In the present case, the following definition is sufficient:

Definition 4 (Multiplier) Let $X$ and $Y$ be Banach spaces whose elements are functions on a common domain of definition $\Lambda$. We say that $Y$ is a multiplier space of $X$ if for every $\rho \in Y$ the pointwise multiplication operator $T_{\rho}$ defined by $\left(T_{\rho} f\right)(x):=\rho(x) f(x)$ for $x \in \Lambda$ is a continuous linear operator from $X$ into itself. In this case, the functions $\rho \in Y$ are called multipliers for $X$.

We give sufficient conditions on when a matrix function is in fact a multiplier on spaces of the type $H^{\varepsilon}(\Omega)^{d}$ for $0<\varepsilon<\frac{1}{2}$, as required in Theorem 1. We do so using Besov spaces $B_{p, q}^{s}(\Omega)$ including those of nonstandard type $p=\infty$. For $0<s<1$ and $q=\infty$, the latter coincide with the Hölder spaces, see [30]. See also [29] and or [27] for the definitions of the Besov space and more.

Clearly, for a matrix-valued function $S: \Omega \rightarrow \mathbb{C}^{d \times d}$ to be a multiplier on $H^{\varepsilon}(\Omega)^{d}$ it is sufficient if every component function $S_{\alpha, \beta}$ for $\alpha, \beta \in\{1, \ldots, d\}$ is a multiplier on $H^{\varepsilon}(\Omega)$ alone. We thus just give conditions for this based on [27,30].

Lemma 1 Let $0<\varepsilon<\frac{1}{2}$ be given. Then, $B_{d(1+\eta) / \varepsilon, 2}^{\varepsilon}(\Omega)$ and $\mathrm{C}^{\sigma}(\Omega)$ are multiplier spaces for $H^{\varepsilon}(\Omega)$ for every $0<\eta \leq \infty$ and $\varepsilon<\sigma<1$.

Proof The results from $[27,30]$ in the following proof are originally stated only for function spaces on $\mathbb{R}^{d}$. The occurring function spaces on $\Omega$ are defined as the restrictions to $\Omega$ of the ones on $\mathbb{R}^{d}$ (cf. Definition 2) which, however, allows to transfer the results from $\mathbb{R}^{d}$ to $\Omega$ by considering functions in the function spaces on $\mathbb{R}^{d}$ whose restriction is the function of interest defined on $\Omega$. For the Hölder spaces, recall that there is the McShane-Whitney extension operator [21].

The multiplier property for $B_{d(1+\eta) / \varepsilon, 2}^{\varepsilon}(\Omega)$ for $0<\eta \leq \infty$ is proven in [27, Thm. 2, Ch. 4.4.4]. Please note here that $H^{s}(\Omega)=F_{2,2}^{s}(\Omega)=B_{2,2}^{s}(\Omega)$. For the case $\eta=\infty$, so $B_{\infty, 2}^{\varepsilon}(\Omega)$ as a multiplier space, see also [27, Ch. 4.7.1]. The assertion for the Hölder spaces now follows from the embedding

$$
B_{\infty, \infty}^{\sigma}(\Omega) \hookrightarrow B_{\infty, 2}^{\varepsilon}(\Omega) \text { for } \sigma>\varepsilon
$$

together with [30, Thm. 4] from which we have

$$
\mathrm{C}^{\sigma}(\Omega) \cong B_{\infty, \infty}^{\sigma}(\Omega) \text { for } 0<\sigma<1 .
$$


Note that the embedding in (5) is not explicitly stated in [30], but follows immediately from the definition of the Besov space there, see [30, p. 77/78], cf. also [27, Ch. 2.2.1].

It is well known that $\sigma$-Hölder functions act as multipliers on $H^{\varepsilon}(\Omega)$ spaces for $\sigma>\varepsilon$, see, e.g., [17, Lem. 2]. We have given another proof as above because we will need the Besov spaces as multipliers in the second application example below in Sect. 8, and the Hölder multiplier result then follows very easily.

Remark 5 Let us point out once more that the conditions in Lemma 1 are merely sufficient and in no way necessary. In fact, due to the embeddings

$$
B_{d(1+\eta) / \varepsilon, 2}^{\varepsilon}(\Omega) \hookrightarrow \mathrm{C}^{\frac{\varepsilon \eta}{1+\eta}}(\Omega) \text { and } B_{\infty, 2}^{\varepsilon}(\Omega) \hookrightarrow B_{\infty, \infty}^{\varepsilon}(\Omega) \cong \mathrm{C}^{\varepsilon}(\Omega),
$$

we see that the given multipliers are all at least Hölder continuous, and $B_{\infty, 2}^{\varepsilon}(\Omega)$ lies in fact between $\mathrm{C}^{\sigma}(\Omega)$ and $\mathrm{C}^{\varepsilon}(\Omega)$ for any $0<\varepsilon<\sigma$, recall (5). But it is also known that the-clearly discontinuous-characteristic function $\chi_{\Xi}$ for an open set $\Xi \subset \Omega$ with locally finite perimeter is also a multiplier on $H^{\varepsilon}(\Omega)$ whenever $|\varepsilon|<\frac{1}{2}$, see [27, p. 214ff]. A general intrinsic characterization of multipliers on $H^{\varepsilon}(\Omega)$ in terms of usual function spaces seems not to be available. We refer to [20,27] and related works, see also [19, Sect. 5].

\section{Proof of the main results}

The proof of Theorem 1 rests on the following fundamental theorem by Šneíberg [31], cf. also [8, Ch. 1.3.5]. For the notions from interpolation theory, we refer to [29, Ch. 1.2, 1.9].

Theorem 2 (Šneǐberg stability theorem) Let $\left(X_{0}, X_{1}\right)$ and $\left(Y_{0}, Y_{1}\right)$ be interpolation couples of Banach spaces, and let $T$ be a continuous linear operator compatible with that interpolation couple. Then, the set

$$
\left\{\theta \in(0,1): T \in \mathcal{L}_{\text {iso }}\left(\left[X_{0}, X_{1}\right]_{\theta} ;\left[Y_{0}, Y_{1}\right]_{\theta}\right)\right\}
$$

is open.

Remark 6 Given a number $\vartheta$ which is an element of the set (6) in Theorem 2, there exist estimates on the size of the open set (6), see [8, Ch. 1.3.5]. These show that the size depends on the operator norms of $T$ as a linear operator from $X_{i}$ to $Y_{i}$ for $i=1,2$, and the operator norm of $T^{-1}$ between $\left[Y_{0}, Y_{1}\right]_{\vartheta}$ and $\left[X_{0}, X_{1}\right]_{\vartheta}$. This is in fact the connection to the claim about uniformity of $\delta$ and the norm of the inverses of $-\nabla \cdot A \nabla+\gamma$ in the main results in Theorem 1 and Corollary 1 .

In order to use Theorem 2, we need to have a suitable interpolation scale at hand. For this, we rely on [9, Ch. 7] from which we cite

Theorem 3 ([9, Thm. 7.1]) Let $F$ be a $(d-1)$-set satisfying $\mathfrak{D} \subseteq F \subseteq \partial \Omega$. Let further $0<\theta<1$ and $\frac{1}{2}<s_{0}, s_{1}<\frac{3}{2}$ and put $s_{\theta}:=(1-\theta) s_{0}+\theta s_{1}$. Then

$$
\left[H_{F}^{s_{0}}(\Omega), H_{F}^{s_{1}}(\Omega)\right]_{\theta}=H_{F}^{s_{\theta}}(\Omega)
$$

and

$$
\left[L^{2}(\Omega), H_{F}^{1}(\Omega)\right]_{\theta}= \begin{cases}H_{F}^{\theta}(\Omega) & \text { if } \theta>\frac{1}{2} \\ H^{\theta}(\Omega) & \text { if } \theta<\frac{1}{2}\end{cases}
$$


Before we prove our main result, we establish a few preparatory lemmas building upon Theorem 3.

Lemma 2 In the situation of Theorem 3, we also have

$$
\left[H_{F}^{-s_{0}}(\Omega), H_{F}^{-s_{1}}(\Omega)\right]_{\theta}=H_{F}^{-s_{\theta}}(\Omega)
$$

and

$$
\left[L^{2}(\Omega), H_{F}^{-1}(\Omega)\right]_{\theta}= \begin{cases}H_{F}^{-\theta}(\Omega) & \text { if } \theta>\frac{1}{2} \\ H^{-\theta}(\Omega) & \text { if } \theta<\frac{1}{2}\end{cases}
$$

Proof This follows quite immediately from the result that the $H_{F}^{s}(\Omega)$ spaces are reflexive [9, Cor. 5.3] and general interpolation duality properties [29, Ch. 1.11.3]. Here, density of $H_{F}^{s_{0}}(\Omega) \cap H_{F}^{s_{1}}(\Omega)=H_{F}^{\max \left(s_{0}, s_{1}\right)}(\Omega)$ in $H_{F}^{s_{0}}(\Omega)$ and $H_{F}^{s_{1}}(\Omega)$ follows from density of $H^{\max \left(s_{0}, s_{1}\right)}\left(\mathbb{R}^{d}\right)$ in $H^{s_{0}}\left(\mathbb{R}^{d}\right)$ and $H^{s_{1}}\left(\mathbb{R}^{d}\right)$ and the characterization $H_{F}^{s}\left(\mathbb{R}^{d}\right)=P_{F} H^{s}\left(\mathbb{R}^{d}\right)$ for a bounded linear projection $P_{F}$ as proven in [9, Cor. 3.5].

Now it only remains to set the stage for the extension of $-\nabla \cdot A \nabla$ to $\mathbb{H}_{D}^{s}(\Omega)$ for $s \neq 1$ before we can give the proof of the main results.

Lemma 3 Let $F$ be $a(d-1)$-set satisfying $\mathfrak{D} \subseteq F \subseteq \partial \Omega$ and let $0<\sigma<\frac{1}{2}$. Then, the weak gradient $\nabla \in \mathcal{L}\left(H_{F}^{1}(\Omega) ; L^{2}(\Omega)^{d}\right)$ maps $H_{F}^{\sigma+1}(\Omega)$ continuously into $H^{\sigma}(\Omega)^{d}$ and admits a unique continuous linear extension $\nabla: H_{F}^{1-\sigma}(\Omega) \rightarrow H^{-\sigma}(\Omega)^{d}$.

Proof The first assertion follows from the corresponding property of $H^{\sigma+1}\left(\mathbb{R}^{d}\right)$ and the definition of the $H_{F}^{\sigma+1}(\Omega)$ spaces. For the second assertion, observe that the distributional gradient $G: L^{2}(\Omega) \rightarrow H_{\partial \Omega}^{-1}(\Omega)^{d}$ is a continuous linear operator, as for all $\varphi \in L^{2}(\Omega)$ we have (recall Proposition 1)

$$
|\langle G \varphi, \xi\rangle|:=\left|-\int_{\Omega} \varphi \operatorname{div} \xi \mathrm{d} x\right| \leq C\|\varphi\|_{L^{2}(\Omega)}\|\xi\|_{H^{1}(\Omega)^{d}} \quad \text { for all } \xi \in \mathrm{C}_{c}^{\infty}(\Omega)^{d} .
$$

Moreover, the distributional gradient $G$ restricted to $H^{1}(\Omega)$ agrees exactly with the weak gradient $\nabla$ on $H^{1}(\Omega)$ per integration by parts and the fundamental lemma of the calculus of variations. Hence, we are able to interpolate the operator (which we agree to call $\nabla$ from now on) which by Theorem 3 and Lemma 2 yields that

$$
\nabla \in \mathcal{L}\left(\left[L^{2}(\Omega), H_{F}^{1}(\Omega)\right]_{1-\sigma} ;\left[H_{\partial \Omega}^{-1}(\Omega)^{d}, L^{2}(\Omega)^{d}\right]_{1-\sigma}\right)=\mathcal{L}\left(H_{F}^{1-\sigma}(\Omega) ; H^{-\sigma}(\Omega)^{d}\right) .
$$

Here, we have used coordinate-wise interpolation in the second component (cf. [8, Cor. 1.3.8]) and the fundamental interpolation property $\left[X_{0}, X_{1}\right]_{\theta}=\left[X_{1}, X_{0}\right]_{1-\theta}$ for any interpolation couple $\left(X_{0}, X_{1}\right)$ and $0<\theta<1$, see [29, Thm. 1.9.3 b)].

We finally prove the main theorem.

Proof (Theorem 1) We had already noted below Proposition 1 that the forms

$$
H_{D_{j}}^{1}(\Omega) \times H_{D_{i}}^{1}(\Omega) \ni(\varphi, \xi) \mapsto\left\langle-\nabla \cdot A^{i, j} \nabla \varphi, \xi\right\rangle:=\left(A^{i, j} \nabla \varphi, \nabla \xi\right)_{L^{2}(\Omega)}
$$

are continuous for $i, j \in\{1, \ldots, n\}$. We extend them to $H_{D_{j}}^{\varepsilon+1}(\Omega) \times H_{D_{i}}^{1-\varepsilon}(\Omega)$ using Lemma 3 as follows, thereby also extending $-\nabla \cdot A \nabla$ to a continuous operator from $\mathbb{H}_{D}^{\varepsilon+1}(\Omega)$ to $\mathbb{H}_{D}^{\varepsilon-1}(\Omega)$, cf. (2): 
Let $i, j \in\{1, \ldots, n\}$ be given and denote by $M_{i, j}$ the norm of $A^{i, j}$ when the latter is considered as a multiplier acting on $H^{\varepsilon}(\Omega)^{d}$. Since $H^{\varepsilon}(\Omega)^{d}$ is dense in $L^{2}(\Omega)^{d}$, we estimate

$$
\begin{array}{r}
\left|\left\langle-\nabla \cdot A^{i, j} \nabla \varphi, \xi\right\rangle\right|=\left|\left(A^{i, j} \nabla \varphi, \nabla \xi\right)_{L^{2}(\Omega)^{d}}\right| \leq\left\|A^{i, j} \nabla \varphi\right\|_{H^{\varepsilon}(\Omega)^{d}}\|\nabla \xi\|_{H^{-\varepsilon}(\Omega)^{d}} \\
\leq M_{i, j}\|\nabla \varphi\|_{H^{\varepsilon}(\Omega)^{d}}\|\nabla \xi\|_{H^{-\varepsilon}(\Omega)^{d}} \leq C M_{i, j}\|\varphi\|_{H_{D_{j}}^{\varepsilon+1}(\Omega)}\|\xi\|_{H_{D_{i}}^{1-\varepsilon}(\Omega)}
\end{array}
$$

for all $\varphi \in H_{D_{j}}^{\varepsilon+1}(\Omega)$ and $\xi \in H_{D_{i}}^{1}(\Omega)$ using Lemma 3. As $H_{D_{i}}^{1}(\Omega)$ is again dense in $H_{D_{i}}^{1-\varepsilon}(\Omega)$, we obtain a unique continuous linear extension of $-\nabla \cdot A^{i, j} \nabla$ to a mapping from $H_{D_{j}}^{\varepsilon+1}(\Omega)$ to $H_{D_{i}}^{\varepsilon-1}(\Omega)$. By definition [see (2)], this also gives a unique continuous linear extension of $-\nabla \cdot A \nabla$ to a mapping from $\mathbb{H}_{D}^{\varepsilon+1}(\Omega)$ to $\mathbb{H}_{D}^{\varepsilon-1}(\Omega)$.

For the extension of $-\nabla \cdot A \nabla$ to an operator $\mathbb{H}_{D}^{1-\varepsilon}(\Omega) \rightarrow \mathbb{H}_{D}^{-1-\varepsilon}(\Omega)$, we observe that taking the conjugate transpose $\left(A^{i, j}\right)^{*}$ of $A^{i, j}$ preserves the multiplier property for $H^{\varepsilon}(\Omega)^{d}$. So analogously to the above we obtain

$$
\left|\left\langle-\nabla \cdot A^{i, j} \nabla \varphi, \xi\right\rangle\right|=\left|\left(\nabla \varphi,\left(A^{i, j}\right)^{*} \nabla \xi\right)_{L^{2}(\Omega)^{d}}\right| \leq C M_{i, j}^{*}\|\varphi\|_{H_{D_{j}}^{1-\varepsilon}(\Omega)}\|\xi\|_{H_{D_{i}}^{1+\varepsilon}(\Omega)}
$$

for $\varphi \in H_{D_{j}}^{1}(\Omega)$ and $\xi \in H_{D_{i}}^{1+\varepsilon}(\Omega)$, where $M_{i, j}^{*}$ denotes the multiplier norm of $\left(A^{i, j}\right)^{*}$. This implies that $-\nabla \cdot A \nabla$ extends to a continuous linear operator from $\mathbb{H}_{D}^{1-\varepsilon}(\Omega)$ to $\mathbb{H}_{D}^{-1-\varepsilon}(\Omega)$. Hence, the operator is compatible with the interpolation couples $\left(\mathbb{H}_{D}^{1+\varepsilon}(\Omega), \mathbb{H}_{D}^{1-\varepsilon}(\Omega)\right)$ and $\left(\mathbb{H}_{D}^{\varepsilon-1}(\Omega), \mathbb{H}_{D}^{-1-\varepsilon}(\Omega)\right)$, and this is then clearly also true for $-\nabla \cdot A \nabla+\gamma$ for any $\gamma \geq 0$.

Now observe that $-\nabla \cdot A \nabla+\gamma \in \mathcal{L}_{\text {iso }}\left(\mathbb{H}_{D}^{1}(\Omega) ; \mathbb{H}_{D}^{-1}(\Omega)\right)$ for $\gamma>\mu$ by the Gårding inequality assumption and the Lax-Milgram lemma, and that

$$
\left[\mathbb{H}_{D}^{1+\varepsilon}(\Omega), \mathbb{H}_{D}^{1-\varepsilon}(\Omega)\right]_{\frac{1}{2}}=\mathbb{H}_{D}^{1}(\Omega) \text { and }\left[\mathbb{H}_{D}^{\varepsilon-1}(\Omega), \mathbb{H}_{D}^{-1-\varepsilon}(\Omega)\right]_{\frac{1}{2}}=\mathbb{H}_{D}^{-1}(\Omega)
$$

due to Theorem 3 and Lemma 2 (and again coordinate-wise interpolation, see [8, Cor. 1.3.8]). But then the stability result of Šnerberg as in Theorem 2 tells us that there exists $0<\delta \leq \varepsilon$ such that we still have $-\nabla \cdot A \nabla+\gamma \in \mathcal{L}_{\text {iso }}\left(\mathbb{H}_{D}^{\theta+1}(\Omega) ; \mathbb{H}_{D}^{\theta-1}(\Omega)\right)$ for all $|\theta|<\delta$. This was the first claim. Note that if $D \neq \emptyset$, then $\gamma=\mu$ is also allowed due to the Poincaré inequality, cf. Remark 1.

For the claimed uniformity of $\delta$ and the norm of the inverse of $-\nabla \cdot A \nabla+\gamma$, we refer to [8, Ch. 1.3.5] and Remark 6.

Proof (Corollary 1) It is a mere reformulation of assertion (3) in Theorem 1 that for every $f \in \mathbb{H}_{D}^{\theta-1}(\Omega)$ there exists a unique $u \in \mathbb{H}_{D}^{\theta+1}(\Omega)$ satisfying the elliptic system equation (4) with $\|u\|_{\mathbb{H}_{D}^{\theta+1}(\Omega)} \leq C\|f\|_{\mathbb{H}_{D}^{\theta-1}(\Omega)}$, where $C$ is independent of $f$.

Now let $\eta \geq 0$ and $p \geq 2$ be such that $\theta \geq \eta+d\left(\frac{1}{2}-\frac{1}{p}\right)$, and consider $j \in\{1, \ldots, n\}$. Then, for every function $U_{j} \in H_{D_{j}}^{\theta+1}\left(\mathbb{R}^{d}\right)$ with the property that $\left(U_{j}\right)_{\mid \Omega}=u_{j}$ we use the well-known (generalized) Sobolev embeddings (cf. [29, Ch. 2.8.1]) as follows:

$$
\left\|u_{j}\right\|_{H_{D_{j}}^{1+\eta, p}(\Omega)} \leq\left\|U_{j}\right\|_{H_{D_{j}}^{1+\eta, p}\left(\mathbb{R}^{d}\right)} \leq C^{\star}\left\|U_{j}\right\|_{H_{D_{j}}^{\theta+1}\left(\mathbb{R}^{d}\right)} .
$$

But this implies that $\left\|u_{j}\right\|_{H_{D_{j}}^{1+\eta, p}(\Omega)} \leq C^{\star}\left\|u_{j}\right\|_{H_{D_{j}}^{\theta+1}(\Omega)}$ and of course accordingly $\|u\|_{\mathbb{H}_{D}^{1+\eta, p}(\Omega)} \leq C^{\star}\|u\|_{\mathbb{H}_{D}^{\theta+1}(\Omega)}$, so the claim follows by observing that if we choose $0<\eta<\theta$, then we are also allowed to choose $p>2$ while still obeying the inequality $\theta \geq \eta+d\left(\frac{1}{2}-\frac{1}{p}\right)$. Uniformity of the collected constants as claimed in the statement of the corollary finally follows immediately from the corresponding assertion in Theorem 1. 


\section{Application: a fracture model}

As an application, we consider a standard phase-field model for brittle fracture as given in [5]. For the following exposition, we consider the formulation given in [23], where the fracture irreversibility is relaxed by a penalty approach. After introduction of a time-discretization, the evolution is given by a sequence of problems associated to each time-step. Namely, for a bounded domain $\Omega \subset \mathbb{R}^{2}$ satisfying Assumption 2, one searches for a (vector-valued) displacement $u \in \mathbb{H}_{D}^{1}(\Omega)$ and a (scalar) phase-field $\phi \in H^{1}(\Omega)$ solving the system of equations

$$
\left.\begin{array}{rl}
(g(\phi) e(u): e(v))= & \ell(v), \\
& \left(\epsilon^{-1}(\phi-1)+(1-\kappa)(\phi e(u): e(u))+\varrho\left[\left(\phi-\phi^{-}\right)^{+}\right]^{3}, \psi\right)_{L^{2}(\Omega)} \\
& +\langle-\nabla \cdot \epsilon \nabla \phi, \psi\rangle=0
\end{array}\right\}
$$

for all $v \in \mathbb{H}_{D}^{1}(\Omega)$ and $\psi \in H^{1}(\Omega)$, with given loads $\ell \in \mathbb{H}_{D}^{\theta_{0}-1}(\Omega)$ for some $\theta_{0}>0, \phi^{-}$ satisfying $0 \leq \phi^{-} \leq 1$, with $0<\kappa \ll \epsilon \ll 1$ and $\varrho>0$ as well as $g(\phi)=(1-\kappa) \phi^{2}+\kappa$, where $e(u)$ and $e(v)$ denotes the symmetric gradient of $u$ and $v$, respectively. It has been shown in [23] that if $\Omega \cup D$ is regular in the sense of Gröger (cf. Remark 3), then this problem admits a Hilbert space solution $(u, \phi) \in \mathbb{H}_{D}^{1}(\Omega) \times H^{1}(\Omega)$ with the additional regularity $u \in \mathbb{W}^{1, p}(\Omega)$ for some $p>2$ and $\phi \in L^{\infty}(\Omega)$; in fact, $0 \leq \phi(x) \leq 1$ holds for almost all $x \in \Omega$.

With the results obtained in this work, we can now show the following improved differentiability result.

Corollary 2 Let $(u, \phi) \in\left(\mathbb{W}^{1, p}(\Omega) \cap \mathbb{H}_{D}^{1}(\Omega)\right) \times\left(H^{1}(\Omega) \cap L^{\infty}(\Omega)\right)$ be the solution of $(7)$ as in [23]. There exists $0<\bar{\theta} \leq \theta_{0}$ such that $(u, \phi)$ admits the additional regularity $u \in$ $\mathbb{H}_{D}^{\theta+1}(\Omega)$ and $\phi \in H^{\theta+1}(\Omega)$ for any $\theta$ satisfying $0<\theta \leq \bar{\theta}$. Moreover, we obtain the estimate

$$
\|u\|_{\mathbb{H}_{D}^{1+\theta}(\Omega)} \leq C\|\ell\|_{\mathbb{H}_{D}^{\theta_{0}-1}(\Omega)}
$$

with a constant $C=C\left(\|\ell\|_{\mathbb{H}_{D}^{-1, p}(\Omega)}^{2}, \varrho, \epsilon\right)$.

Proof Slightly rewriting the second equation in (7), we see that $\phi$ satisfies

$$
\left(-\nabla \cdot \epsilon \nabla+\epsilon^{-1}\right) \phi=\epsilon^{-1}+(\kappa-1)(\phi e(u): e(u))-\varrho\left[\left(\phi-\phi^{-}\right)^{+}\right]^{3} \text { in } H^{-1}(\Omega) .
$$

By the regularity $\phi \in L^{\infty}(\Omega)$ and $u \in \mathbb{W}^{1, p}(\Omega)$, it is clear that the right hand side is in fact an element of $L^{p / 2}(\Omega)$. Consequently, by Sobolev embedding, there exists some $\vartheta>0$ such that it is an element of $H^{\vartheta-1}(\Omega)$. Theorem 1 then shows that we have $\phi \in H^{\theta+1}(\Omega)$ for all $0<\theta \leq \bar{\vartheta}$ for some $\bar{\vartheta} \leq \vartheta$, and standard Sobolev embedding theorems assert that $\phi \in \mathrm{C}^{\sigma}(\Omega)$ for $\sigma=1+\theta-\frac{2}{p}$. Moreover, by [23, Corollary 4.2], we have that $\|\phi e(u): e(u)\|_{L^{p / 2}(\Omega)} \leq c\|\ell\|_{\mathbb{H}_{D}^{-1, p}(\Omega)}^{2}$ for some constant $c \geq 0$, and thus

$$
\|\phi\|_{C^{\sigma}(\Omega)} \leq c\left(\|\ell\|_{\mathbb{H}_{D}^{-1, p}(\Omega)}^{2}+\varrho+\epsilon^{-1}\right) .
$$

But then, by definition, $g(\phi) \in \mathrm{C}^{\sigma}(\Omega)$ too and Lemma 1 (iii) shows that this is indeed a multiplier on $\mathbb{H}^{\theta}(\Omega)$. Now another application of Theorem 1 to the equation

$$
(g(\phi) e(u): e(v))=\ell(v) \text { for all } v \in \mathbb{H}_{D}^{1}(\Omega)
$$


yields the claimed regularity. For the stability estimate, we utilize the above bound on $\|\phi\|_{C^{\sigma}(\Omega)}$ together with the uniformity assertion in Corollary 1.

Remark 7 In the case where the irreversibility of the fracture is not relaxed via a penalization approach, the equation for $\phi$ becomes an obstacle problem where the term involving $\varrho([(\phi-$ $\left.\left.\phi^{-}\right)^{+}\right]^{3}$ is replaced by the requirement $\phi \leq \phi^{-}$. If the domain is sufficiently regular, then classical $\mathrm{W}^{2, p / 2}(\Omega)$-regularity of the obstacle problem, i.e., $\phi \in \mathrm{W}^{2, p / 2}(\Omega)$ as long as $\phi^{-} \in$ $\mathrm{W}^{2, p / 2}(\Omega)$, can be used to show that $\phi$ is again a multiplier (see, e.g., [6, Corollary II.3]).

This improved regularity result is very valuable in the numerical analysis of the fracture processes described above, as the improved regularity $\mathbb{H}_{D}^{1+\theta}(\Omega)$ allows to quantify bestapproximation errors in $\mathbb{H}_{D}^{1}(\Omega)$ in terms of the discretization fineness. Similar known results for improved regularity in the $\mathbb{H}_{D}^{1, p}(\Omega)$ scale do not allow to do so. We note that due to the numerical background, the full potential of the assumptions on the spatial set $\Omega$ and its boundary parts is not required for this example. However, there is no comparable result known to the authors so far which allowed to treat the system (7) in a non-smooth Lipschitz setting for $\Omega$ with mixed boundary conditions, e.g., in the setting of Gröger.

\section{Application: quasilinear equations}

We give another possible application for Theorem 1. Let us consider a single abstract parabolic quasilinear evolution equation of the form

$$
u^{\prime}(t)-\nabla \cdot A(u(t)) \nabla u(t)=F(u(t)), \quad u(0)=u_{0} .
$$

For this exposition, we assume that the (nonlinear) functions $A$ and $F$ and the initial value $u_{0}$ are suitably regular. Suppose we want to treat (8) in the space $H_{D}^{\theta-1}(\Omega)$ for $\Omega \subset \mathbb{R}^{2}$. (We give a motivation why this is interesting below Remark 8.)

A possible way to do so are the abstract frameworks of Amann [2] and Prüss [25, Thm. 3.1] based on maximal regularity techniques. One of the most critical points to verify for this is that there is a space $\mathcal{D}_{\theta}$ such that $-\nabla \cdot A(u) \nabla$ is a continuous linear operator from $\mathcal{D}_{\theta}$ to $H_{D}^{\theta-1}(\Omega)$ for all $u$ from the trace or interpolation space $\left(\mathcal{D}_{\theta}, H_{D}^{\theta-1}(\Omega)\right)_{1 / p, p}$ for some $1<p<\infty$. Here, our main Theorem 1 comes into play: If we are able to show that $u \in\left(H_{D}^{1+\theta}(\Omega), H_{D}^{\theta-1}(\Omega)\right)_{1 / p, p}$ implies that $A(u)$ is a suitable multiplier on $H^{\varepsilon}(\Omega)^{2}$ for some $\theta<\varepsilon<\frac{1}{2}$, then the theorem shows that $\mathcal{D}_{\theta}=H_{D}^{1+\theta}(\Omega)$ indeed does the job.

Indeed, using the recent results in [4] which hold true under our general assumptions on $\Omega$, one may show by interpolation techniques that

$$
\left(H_{D}^{1+\theta}(\Omega), H_{D}^{\theta-1}(\Omega)\right)_{\frac{1}{p}, p} \hookrightarrow B_{\frac{d}{\varepsilon}(1+\eta), 2}^{\varepsilon}(\Omega)
$$

if

$$
\theta<\varepsilon<\frac{1+\eta}{\eta} \theta \text { and } p>\left(1+\theta-\varepsilon \frac{\eta}{1+\eta}-\frac{d}{2}\right)^{-1}
$$

with $\eta>\theta$, but finite. If we then further suppose that $A$ is sufficiently regular to pass the regularity of $u$ to $A(u)$, then Theorem 1 and Lemma 1 tell us that $-\nabla \cdot A(u) \nabla$ is indeed a continuous linear operator from $H_{D}^{1+\theta}(\Omega)$ to $H_{D}^{\theta-1}(\Omega)$ for every $u \in\left(H_{D}^{1+\theta}(\Omega), H_{D}^{\theta-1}(\Omega)\right)_{1 / p, p}$ for $p$ as given above. The condition on $p$ shows why we needed to restrict ourselves to $d=2$ here, as $1-\frac{d}{2}+\theta<0$ for $d \geq 3$ due to $\theta<\frac{1}{2}$. 
So, while there are still quite formidable assumptions left to establish for the frameworks in $[2,25]$ to completely treat the quasilinear equation in $H_{D}^{\theta-1}(\Omega)$, Theorem 1 can be used as a starting point to do so as explained above.

Remark 8 Let us point out that the space $B_{\infty, 2}^{\varepsilon}(\Omega)$, so $\eta=\infty$ in $B_{d(1+\eta) / \varepsilon, 2}^{\varepsilon}(\Omega)$, is not suitable as a multiplier space here since $H_{D}^{1+\theta}(\Omega) \hookrightarrow B_{\infty, 2}^{\theta}(\Omega)$ and the smoothness order in the Besov space cannot be improved ([27, Thm. 2.2.3]). This is already insufficient for the multiplier property on $H^{\varepsilon}(\Omega)$ due to the requirement $\varepsilon>\theta$ and will not improve by interpolation with $H_{D}^{\theta-1}(\Omega)$. In this sense, we really need $B_{d(1+\eta) / \varepsilon, 2}^{\varepsilon}(\Omega)$ with finite $\eta$ as a multiplier space for $H^{\varepsilon}(\Omega)$ here.

Finally, let us briefly explain why it is of interest to treat the quasilinear equation in $H_{D}^{1+\theta}(\Omega)$ in space dimension $d=2$ : Consider a bounded function $u:[0, T] \rightarrow$ $\left(H_{D}^{1+\theta}(\Omega), H_{D}^{\theta-1}(\Omega)\right)_{1 / p, p}$. For $p$ suitably large, we expect that $|\nabla u(t)|^{2}$ admits enough integrability to give rise to an element of $H_{D}^{\theta-1}(\Omega)$, since then the interpolation space is sufficiently close to $H_{D}^{1+\theta}(\Omega)$ to still embed into a space of type $H_{D}^{1, p}(\Omega)$ with $p>d=2$, as in the proof of Corollary 1. Hence, treating the quasilinear equation in $H_{D}^{\theta-1}(\Omega)$ in space dimension $d=2$ would allow to incorporate quadratic gradient terms of $u(t)$ in $F(u(t))$ which are of practical relevance, in this very general geometric framework. Here, we have assumed $u$ to be bounded so that there occurs no loss of time integrability over the finite time interval $[0, T]$ between $u(\cdot)$ in the interpolation space and $|\nabla u(\cdot)|^{2}$ in $H_{D}^{\theta-1}(\Omega)$. To achieve this boundedness in the maximal regularity setting, we need to consider $u$ with values in the interpolation space.

Acknowledgements The authors express their gratitude to the anonymous referee for useful comments and Joachim Rehberg (WIAS Berlin) for valuable discussions. Hannes Meinlschmidt is grateful for support of his former institution TU Darmstadt; Winnifried Wollner acknowledges funding by the DFG priority program 1962.

\section{References}

1. Amann, H.: Maximal regularity for nonautonomous evolution equations. Adv. Nonlinear Stud. 4(4), 417-430 (2004)

2. Amann, H.: Quasilinear parabolic problems via maximal regularity. Adv. Differ. Equ. 10(10), 1081-1110 (2005)

3. Auscher, P., Badr, N., Haller-Dintelmann, R., Rehberg, J.: The square root problem for second-order, divergence form operators with mixed boundary conditions on $L^{p}$. J. Evol. Equ. 15(1), 165-208 (2014). https://doi.org/10.1007/s00028-014-0255-1

4. Bechtel, S., Egert, M.: Interpolation theory for Sobolev functions with partially vanishing trace on irregular open sets. Preprint arXiv:1807.02293

5. Bourdin, B., Francfort, G.A., Marigo, J.J.: The variational approach to fracture. J. Elast. 91(1-3), 5-148 (2008). https://doi.org/10.1007/s10659-007-9107-3

6. Brezis, H., Stampacchia, G.: Sur la régularité de la solution d'inéquations elliptiques. Bull. Soc. Math. Fr 96, 153-180 (1968)

7. Ciarlet, P.G.: Mathematical Elasticity: Three-Dimensional Elasticity. No. Bd. 1 in Studies in Mathematics and Its Applications. North-Holland, Amsterdam (1988)

8. Egert, M.: On Kato's conjecture and mixed boundary conditions. Sierke. Ph.D. Thesis, TU Darmstadt (2015)

9. Egert, M., Haller-Dintelmann, R., Tolksdorf, P.: The Kato square root problem for mixed boundary conditions. J. Funct. Anal. 267(5), 1419-1461 (2014). https://doi.org/10.1016/j.jfa.2014.06.003

10. Giaquinta, M., Martinazzi, L.: An Introduction to the Regularity Theory for Elliptic Systems, Harmonic Maps and Minimal Graphs, Appunti. Scuola Normale Superiore di Pisa (Nuova Serie) [Lecture Notes. 
Scuola Normale Superiore di Pisa (New Series)], vol. 11. Springer Nature (2012). https://doi.org/10. 1007/978-88-7642-443-4

11. Gilbarg, D., Trudinger, N.S.: Elliptic Partial Differential Equations of Second Order, Grundlehren der mathematischen Wissenschaften, vol. 224, revised 3 edn. Springer, Berlin (2001)

12. Grisvard, P.: Elliptic Problems in Nonsmooth Domains. Monographs and Studies in Mathematics, 1st edn. Pitman, Boston (1985)

13. Gröger, K.: A $W^{1, p}$-estimate for solutions to mixed boundary value problems for second order elliptic differential equations. Math. Ann. 283(4), 679-687 (1989). https://doi.org/10.1007/BF01442860

14. Gröger, K., Rehberg, J.: Resolvent estimates in $W^{-1, p}$ for second order elliptic differential operators in case of mixed boundary conditions. Math. Ann. 285(1), 105-113 (1989). https://doi.org/10.1007/ BF01442675

15. Herzog, R., Meyer, C., Wachsmuth, G.: Integrability of displacement and stresses in linear and nonlinear elasticity with mixed boundary conditions. J. Math. Anal. Appl. 382(2), 802-813 (2011). https://doi.org/ 10.1016/j.jmaa.2011.04.074

16. Horgan, C.O.: Korn's inequalities and their applications in continuum mechanics. SIAM Rev. 37(4), 491-511 (1995). https://doi.org/10.1137/1037123

17. Jochmann, F.: An $H^{s}$-regularity result for the gradient of solutions to elliptic equations with mixed boundary conditions. J. Math. Anal. Appl. 238(2), 429-450 (1999). https://doi.org/10.1006/jmaa.1999. 6518

18. Jonsson, A., Wallin, H.: Function spaces on subsets of $R^{n}$. Math. Rep. 2(1), 1-221 (1984)

19. Marschall, J.: Nonregular pseudo-differential operators. Z. Anal. Anwendungen 15(1), 109-148 (1996). https://doi.org/10.4171/zaa/691

20. Maz'ya, V., Shaposhnikova, T.O.: Theory of Sobolev Multipliers, Grundlehren der mathematischen Wissenschaften, vol. 337. Springer, Berlin (2009). https://doi.org/10.1007/978-3-540-69492-2

21. McShane, E.J.: Extension of range of functions. Bull. Am. Math. Soc. 40(12), 837-842 (1934)

22. Neff, P., Pompe, W.: Counterexamples in the theory of coerciveness for linear elliptic systems related to generalizations of Korn's second inequality. Z. Angew. Math. Mech. 94(9), 784-790 (2014). https://doi. org/10.1002/zamm.201300059

23. Neitzel, I., Wick, T., Wollner, W.: An optimal control problem governed by a regularized phase-field fracture propagation model. SIAM J. Control Optim. 55(4), 2271-2288 (2017). https://doi.org/10.1137/ $16 \mathrm{M} 1062375$

24. Oden, J.T.: Existence theorems for a class of problems in nonlinear elasticity. J. Math. Anal. Appl. 69(1), 51-83 (1979). https://doi.org/10.1016/0022-247X(79)90178-1

25. Prüss, J.: Maximal regularity for evolution equations in $L^{p}$-spaces. Conf. Semin. Mat. Univ. Bari 285, 1-39 (2002)

26. Prüss, J., Schnaubelt, R.: Solvability and maximal regularity of parabolic evolution equations with coefficients continuous in time. J. Math. Anal. Appl. 256(2), 405-430 (2001). https://doi.org/10.1006/jmaa. 2000.7247

27. Runst, T., Sickel, W.: Sobolev Space of Fractional Order, Nemytskij Operators, and Nonlinear Partial Differential Equations, Nonlinear Analysis and Applications, vol. 3. Walter de Gruyter, Berlin (1996)

28. Serre, D.: Second-order initial-boundary value problems of variational type. J. Funct. Anal. 236(2), 409446 (2006). https://doi.org/10.1016/j.jfa.2006.02.020

29. Triebel, H.: Interpolation Theory, Function Spaces, Differential Operators, North-Holland Mathematical Library, vol. 18. North-Holland Publishing Co., Amsterdam (1978)

30. Triebel, H.: On spaces of $B_{\infty, q}^{s}$ type and $\mathfrak{C}^{s}$ type. Math. Nachr. 85, 75-90 (1978)

31. Šněberg, I.J.: Spectral properties of linear operators in interpolation families of Banach spaces. Mat. Issled 9(2(32)), 214-229, 254-255 (1974)

32. Zhang, K.: On coercivity and regularity for linear elliptic systems. Calc. Var. Partial Differ. Equ. 40(1-2), 65-97 (2011). https://doi.org/10.1007/s00526-010-0334-2

Publisher's Note Springer Nature remains neutral with regard to jurisdictional claims in published maps and institutional affiliations. 